\title{
The Inherent Relationship between Traditional Chinese Medicine (TCM) and Hypnotic Rituals of Awareness Under Conscious Hypnosis (AUCHC) Method
}

\author{
Ali Özden Öztürk* and Gizemnur Öztürk \\ Society of Medical Hypnosis (THD) Istanbul, Turkey
}

Submission: January 16, 2018; Published: February 07, 2018

*Corresponding author: Ali Özden Öztürk, MD, Koşuyolu Cad. As Çamlık Sitesi B1 Blok No: 77 Daire: 7 Kadıköy 34718 İstanbul/ Turkey, Tel: 0090 54144766 73/ 009021632696 00; Fax: 009021632656 00; Email: auchozturk@gmail.com

Abstract

Introduction: Hypnosis and acupuncture has been considered as effective for the treatment of various illnesses. On the other hand, the integrative use of these two therapies simultaneously is not common in general practice. Hence, this paper is aimed at elaborating the inherent relationship between Traditional Chinese Medicine (TCM) and hypnotic rituals of a Conscious Hypnosis method named "Awareness Under Conscious Hypnosis (AUCHC)" to shed some light on the integrative use of hypnosis and acupuncture.

Methods: The relationship between TCM and AUCH (C) hypnotic rituals was analysed by mapping TCM acupoints and meridians coinciding with the various body areas induced spontaneously via "hand passes, touchings and tapings" as an intrinsic part of hypnotic treatment protocol of AUCH@ Method.

Results: This mapping has systematically illustrated that TCM acupoints and meridians have an inherent relationship with hypnotic rituals applied intrinsically on various body parts during hypnotic induction and autohypnosis steps of AUCHC.

Conclusion: As a consequence, it is worth to pay regard to the inherent relationship between TCM and hypnotic rituals of AUCH@ by taking into consideration the efficient application of AUCH@ Method in surgical operations as the sole anesthetic for pain relief, and also for patient care during pre-, post- and intra-operative periods besides its various competent applications in different fields of medicine. On the other side, further research is necessary to understand the mechanism and rationale behind the inherent relationship between TCM and hypnotic rituals of AUCHC Method, and thus to gain further insight into the integrative use of hypnosis and acupuncture.

Keywords: Traditional Chinese Medicine (TCM); Acupuncture; Awareness Under Conscious Hypnosis (AUCHC); Medical Hypnosis; Autohypnosis; Integrative Medicine

\section{Introduction}

Hypnosis has gained in recognition as an effective treatment for many psychological and physical ailments, and has a wide application area [1-6]. Correspondingly, acupuncture has been acknowledged as valuable for treating various health conditions [7-9]. On the other hand, these two complementary and integrative medicine therapies are commonly administered separately in general practice, while there are views elaborating the simultaneous administration of both hypnosis and acupuncture (i.e. hypno-acupuncture), and supporting the synergism hypothesis between hypnosis and acupuncture when applied together $[7,10,11]$. Hence, with this study, we aim to elaborate the inherent relationship between Traditional
Chinese Medicine (TCM) $[8,9,12,13]$ and the hypnotic rituals of a conscious hypnosis method named "Awareness Under Conscious Hypnosis (AUCHC)" [14-16], and thus to examine further the feasibility of the integrative use of hypnosis and acupuncture.

\section{Methods}

The inherent relationship between TCM and hypnotic rituals of AUCH@ $\subseteq$ was analysed and illustrated via mapping TCM meridians and points coinciding with the various body areas induced spontaneously during the treatment protocol of AUCH@ Method. AUCH $\subset$ is used as the main method as described in detail in the following lines. The hypnotic rituals are applied 
in induction step and also with the autohypnotic key used in autohypnosis step of AUCH@ Method. Also, the mapping is illustrated via describing some of the basic hypnotic rituals of AUCH(C Method. While showing TCM meridians and points on the map, "Teaching Atlas of Acupuncture: Channels and Points" [12] and also "The Pictorial Atlas of Acupuncture: An Illustrated Manual of Acupuncture Points" [13] are used as the reference sources.

\section{Awareness Under Conscious Hypnosis (AUCH@) Method}

AUCHC is a Conscious Hypnosis method established by Dr. Hüsnü İsmet Öztürk in 1951 and in which the person under hypnosis is fully conscious and awake during the whole hypnotic process. AUCH@ Method can be applied in many different fields of medicine for complementary and integrative healing purposes. It has a holistic view of human that has many different value and belief systems and sociocultural norms, thus provides an inherently and systematically integrative therapeutic approach. This leads to the possibility of using other medical and psychological disciplines as a part of the transcultural and interdisciplinary methodology of AUCHC. In this respect, TCM has an inherent relationship with AUCH@ Method since the body parts induced spontaneously during AUCH (C hypnotic treatment protocol coincide considerably with the acupuncture points and meridians of TCM [15].

\section{Hypnotic treatment protocol of AUCHC}

AUCH(C) is a state of consciousness aiming to make changes in attention, perception, memory, emotions and senses of the patient in order to regulate, maintain and improve patient's psychological, physiological and social wellbeing. For this purpose, AUCHC uses a treatment protocol consisting of three steps: "1) MAYA(C (Making Acceptance with Your Awareness), 2) Induction and 3) Autohypnosis" [14] (Table1).

Table 1: Hypnotic Treatment Protocol of Awareness Under Conscious Hypnosis (AUCHO) Method.

\begin{tabular}{|c|c|}
\hline Main Steps of AUCH@ & Hypnotic Treatment Protocol of AUCH (C) \\
\hline $\begin{array}{c}\text { 1) MAYAC } \\
\text { (Making Acceptance } \\
\text { with Your Awareness) }\end{array}$ & $\begin{array}{l}\text { To listen to the patient; observation and consultation; } \\
\text { To accept the patient to the therapy; } \\
\text { To establish rapport (attachment); } \\
\text { To shape the therapy plan according to the needs and expectations of the patient; } \\
\text { To use stories or metaphors for giving the patient positive aspects of conflicts and a wider perspective; } \\
\text { To give general info regarding hypnosis, AUCH@ and treatment tools/methodology (Warming up period); } \\
\text { To understand basic \& actual capacities and conflicts of the patient; } \\
\text { To improve rapport; to answer patient's all questions regarding treatment method (Cooling down period) } \\
\text { To illustrate AUCH (C) application videos to encourage the patient for the treatment and the utilization of self- } \\
\text { help capabilities; } \\
\text { To take the consent of the patient. }\end{array}$ \\
\hline 2) Induction & $\begin{array}{l}\text { To use hypnotic suggestions, induction techniques ("Eye-to-Eye Fixation and Giving Suggestions Technique" as } \\
\text { the main induction technique) and hypnotic rituals according to the needs and expectations of the patient in } \\
\text { order to solve conflicts and problems of the patient; } \\
\text { To develop or differentiate patient's capacities while increasing communication capabilities (differentiation); } \\
\text { To use collective and personal myths to increase the effectiveness of the therapy; } \\
\text { To apply "hand passes, touchings and tapings" as a part of hypnotic rituals intrinsic to AUCH@ Method on and } \\
\text { over head and body parts coinciding considerably with acupuncture points, meridians and zones. }\end{array}$ \\
\hline 3) Autohypnosis & $\begin{array}{l}\text { To give the patient the ability to use autohypnosis whenever/ wherever needed but under certain conditions } \\
\text { designated by the practitioner in order to achieve, reinforce and maintain the therapeutic aims; } \\
\text { To lead the patient to determine and achieve future goals and roles by using self-help capacities (detachment). } \\
\text { If the aimed therapeutic results are reached, to teach the patient the use of "autohypnotic key" specific to } \\
\text { AUCH@ method, "covering the thumb with other four fingers in a fist", which spontaneously induces hand } \\
\text { areas coinciding substantially with acupuncture points, meridians and zones. }\end{array}$ \\
\hline
\end{tabular}

The table shows the 3 steps of AUCH@ Method: Making Acceptance with Your Awareness (MAYA@), Induction and Autohypnosis, all of which constitute the hypnotic treatment protocol of AUCH@. 
Results

\section{The mapping based on the inherent relationship between TCM and the hypnotic rituals applied during hypnotic induction step}

Various hypnotic induction techniques can be used to induce hypnosis in Induction Step. On the other hand, in AUCH@ method, "Eye-to-Eye Fixation and Giving Suggestions Technique" is the main induction technique during whole induction period. This induction technique is unique to AUCH@ method due to the active use of hypnotic rituals including "hand passes, touchings and tapings" on and over head and body parts, and also based mainly on the hypnotic suggestions given to the patient.

AUCH(C) Method has 41 main hypnotic rituals that can be applied during Induction Step, but in this study only three of them will be mentioned. To prevent confusion, they will be numbered respectively, but this numbering does not necessarily mean they need to be applied subsequently in practice.

The starting position of the "Eye-to-Eye Fixation and Giving Suggestions Technique" and the first hypnotic ritual is settled by the practitioner as below (Figure $1 \& 2$ ):

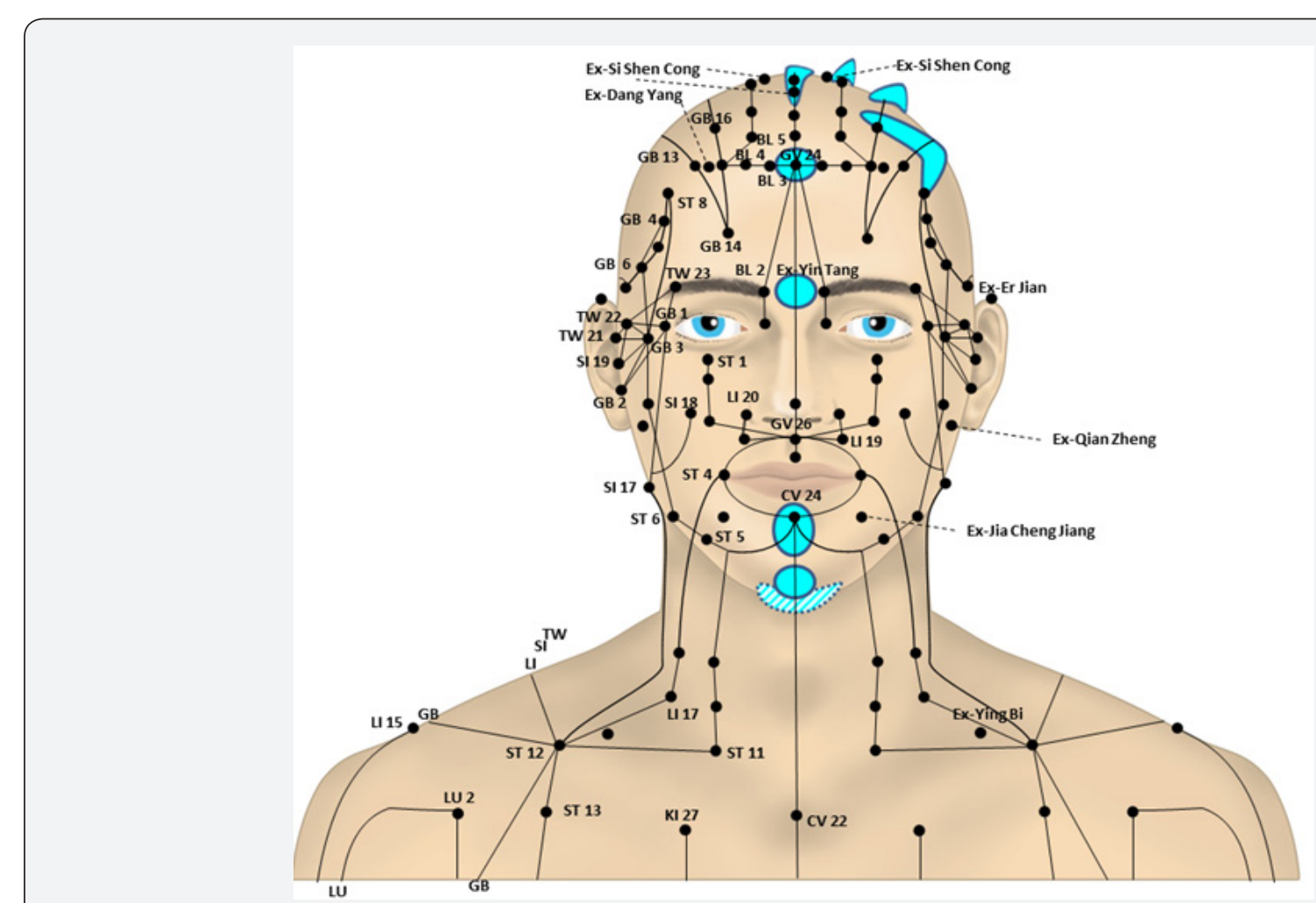

Figure 1: The main positions of the fingers during first hypnotic ritual- anterior aspect.

(Note: The figure demonstrates TCM acupoints and meridians. The positions of the fingers are illustrated via coloured areas. The area with dashed line shows the position of the fingers on the neck.

LI: Large Intestine; ST: Stomach; SI: Small Intestine; BL: Bladder; TW: Triple Warmer; GB: Gallbladder, GV: Governing Vessel; CV: Conception Vessel).

i. Left thumb is located covering Governing Vessel (GV) 24 (the intersection point of the GV, Bladder (BL) and Stomach (ST) Meridians).

ii. Left index finger is positioned covering ST8 (the intersection point of the ST, Gallbladder (GB) and Yang Wei Meridians).

iii. Left middle and ring fingers are located over the area including GB and BL Meridians.

iv. Left little finger is placed on the area covering GV20 (the intersection point of the GV, BL and Liver (LR) Meridians) and Exsi Shen Cong.

v. Right thumb of the practitioner holds the chin of the patient around the intersection point of the mandible base and
Conception Vessel (CV) Meridian.

vi. Right index and middle fingers rest on the area around CV23 (the intersection point of the CV and Yin Wei Mai). This area on the neck also covers Ex-Shang Lianquan.

After settling the starting position of this technique, the practitioner looks through the eyes of the patient from a distance of approximately 20-25 cm while pressing downwards the left thumb on the sagittal plane slowly from GV24 area towards glabella following GV meridian. The left thumb stops on the area covering Ex-Yin Tang when the patient's eyes get closed simultaneously. At the same time, the practitioner moves the right thumb upwards with a gentle pressure on the sagittal plane towards mentolabial groove and stops on the area including 
CV24 (the intersection point of the CV, Large Intestine (LI) and ST Meridians).

The second hypnotic ritual that can be used during the application of the "Eye-to-Eye Fixation and Giving Suggestions Technique" is applied on either side of the face like this (Figure 3):

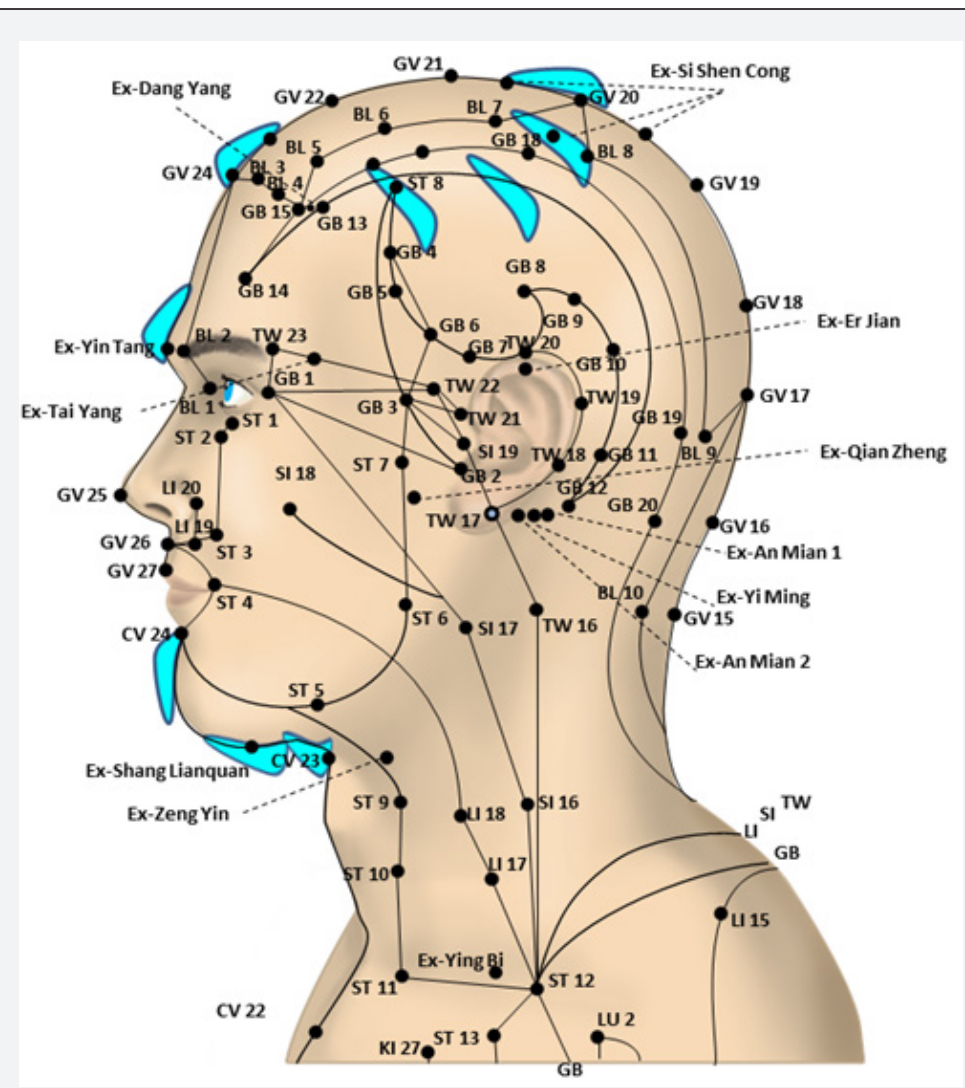

Figure 2: The main positions of the fingers during first hypnotic ritual- lateral aspect.

(Note: The figure demonstrates TCM acupoints and meridians. The positions of the fingers are illustrated via coloured areas.

LI: Large Intestine; ST: Stomach; SI: Small Intestine; BL: Bladder; TW: Triple Warmer; GB: Gallbladder, GV: Governing Vessel; CV: Conception Vessel)

i. Each thumb of the practitioner is placed on either side of the median line between eyebrows including the area between BL2 and GV.

ii. Each index finger is put right in front of the upper ear muscle covering GB7 (the intersection point of the GB and BL Meridians).

iii. Each middle finger is positioned in front of tragus, on the area covering GB2, Small Intestine (SI) 19 (the intersection point of the SI, Triple Warmer (TW) and GB Meridians), and TW21.

iv. Each ring finger is positioned on the area including TW17 (the intersection point of the TW and GB Meridians).

v. Each little finger is located on the area covering ST6 (the intersection point of the ST and GB Meridians).

This position of the hands of the practitioner is followed by light micro circular passes made in front of and top of each ear with the other fingers while each thumb is fixed between the eyebrows.

The third hypnotic ritual initiates with the positioning of the practitioner's hands on either side of the patient's face as below (Figure 4):

i. Each thumb is put in front of tragus on the area including GB2, SI19 and TW21 where GB, TW, ST and SI meridians come together.

ii. Each index finger is placed superior to the apex of the ear and over the triangle area having GB7, GB8 (the intersection point of the GB and BL Meridians) and TW20 (the intersection point of the GB and TW Meridians) as its corners.

iii. Each middle finger and ring finger is placed behind the auricle while middle finger is located around GB10 (the intersection point of the GB and BL Meridians) and TW19, and ring finger is positioned covering GB11 (the intersection point of the GB and BL Meridians).

iv. Each little finger is located on the mastoid process around GB12 (the intersection point of the GB and BL Meridians).

Then, the practitioner makes circular hand passes on each temporal region with the tips of other fingers while each thumb is fixed on its initial position, i.e. in front of tragus. These hand 
passes on each side of the patient's head starts from the upper ear muscle and goes towards mastoid process following the path behind the auricle. Along this path, GB and TW meridians were passed by fingertips with a gentle pressure. When each index finger reaches to the lobule of the ear and passes over the area including GB12 and TW17, each thump makes a lateral rotation and turns upwards without touching the patient's body. Then, with a light touch, the dorsa-distal surfaces of the other four fingers on each side slide further down to the neck, pass over sternocleidomastoid muscle then clavicle, and reach to the neck triangle which is located above the clavicle between the front edge of the trapezius muscle and the lateral part of the sternocleidomastoid muscle. These hand passes finally end on the hand fingertips of the patient on either side, after passing over the anterior, lateral, posterior and/or medial surface of each arm depending on the position of the patient.

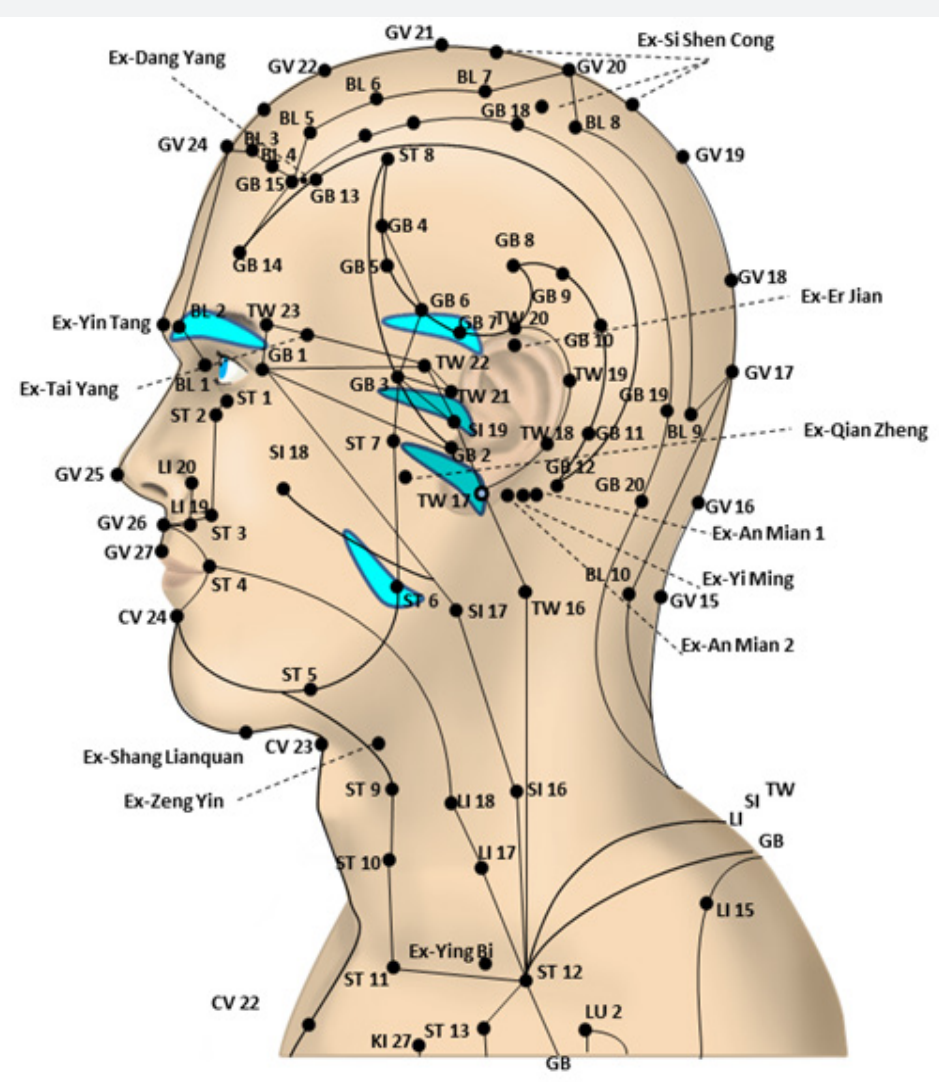

Figure 3: The main positions of the fingers during second hypnotic ritual.

(Note: The figure demonstrates TCM acupoints and meridians. The positions of the fingers are illustrated via coloured areas. TW17 is demonstrated with a ring since it is behind the ear lobe.

LI: Large Intestine; ST: Stomach; SI: Small Intestine; BL: Bladder; TW: Triple Warmer; GB: Gallbladder, GV: Governing Vessel; CV: Conception Vessel)

As mentioned before, these hypnotic rituals are among the 41 basic hypnotic rituals used during the treatment process of AUCHC method. On the other hand, additional micro hand passes, touchings and tapings, also hypnotic rituals can be applied on and over the upper and lower body during or apart from these hypnotic rituals depending on the treatment plan.

It is also important to note that all the hypnotic rituals including hand passes, touchings and tapings are accompanied by hypnotic suggestions given simultaneously during whole induction period according to the needs and expectations of the patient; and that these hypnotic suggestions are the main and key therapeutic tool in AUCH(C) method. The suggestions are designed in accordance with the diagnosis made according to the observations and information gathered during MAYA(C) step. Also, if needed, the suggestions can be reshaped or new suggestions can be added to the existing ones during treatment period.

\section{The mapping based on the inherent relationship between TCM and the hypnotic ritual applied during autohypnosis step}

If the treatment applied becomes successful providing the desired outcomes, autohypnosis is taught to the patient as the third step of AUCH@ hypnotic treatment protocol. So, the patient can achieve, maintain and reinforce the awareness, well-being and self-help ability gained during the treatment whenever and wherever needed as soon as it is within the borders specified by the practitioner. Although it is possible to use different autohypnosis techniques, AUCH $(\mathrm{C}$ method uses a unique 
autohypnotic key, a hand gesture made with the dominant hand, to initiate autohypnosis process. This autohypnotic key, which is covering the thumb with other four fingers in a fist, is intrinsic to AUCH(C) method since the hand area induced spontaneously with the hypnotic ritual applied during autohypnosis step coincide considerably with TCM acupuncture points and meridians as illustrated in Figure 5 and 6. On the other hand, it should be noted that the main therapeutic tool used during autohypnosis is the set of hypnotic suggestions given by the practitioner to the patient according to the treatment plan in consideration of the patient's needs and expectations.

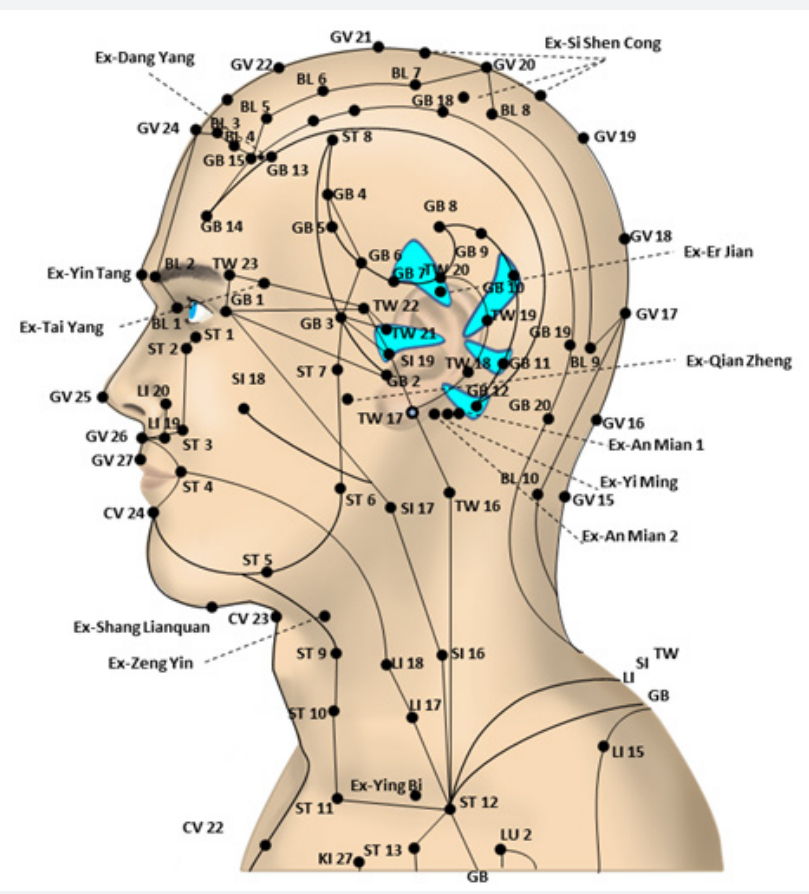

Figure 4: The main positions of the fingers during third hypnotic ritual.

(Note: The figure demonstrates TCM acupoints and meridians. The positions of the fingers are illustrated via coloured areas. TW17 is demonstrated with a ring since it is behind the ear lobe.

LI: Large Intestine; ST: Stomach; SI: Small Intestine; BL: Bladder; TW: Triple Warmer; GB: Gallbladder, GV: Governing Vessel; CV: Conception Vessel)

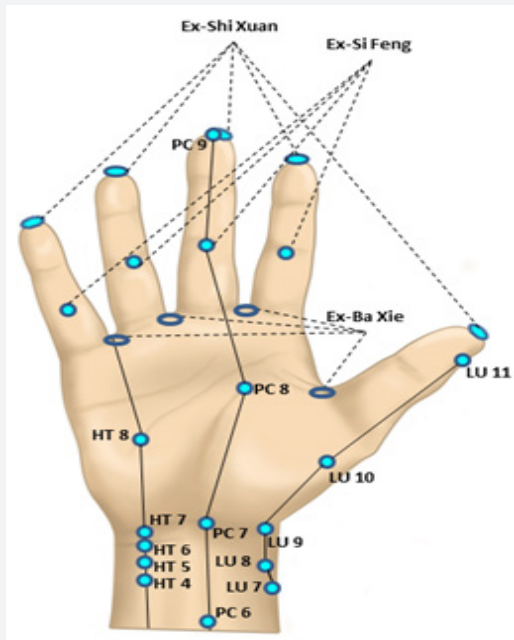

Figure 5: TTCM Acupuncture points and meridians on the palmar surface of the hand.

(Note: The acupoints on the dorsal surface of the hand are demonstrated with a ring.

LU: Lung; HT: Heart; PC: Pericardium) 


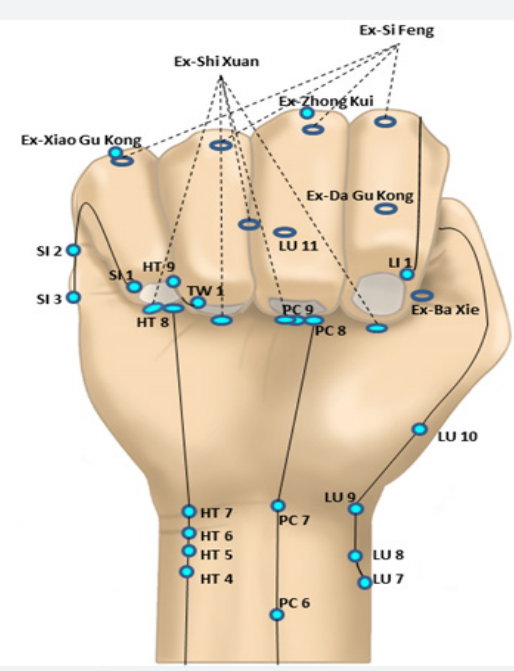

Figure 6: TCM Acupuncture points and meridians on hand and Autohypnotic Key Sign of AUCH○ Method.

(Note: The acupoints under the fingers are demonstrated with a ring.

LU: Lung; LI: Large Intestine; HT: Heart; SI: Small Intestine; PC: Pericardium; TW: Triple Warmer)

\section{Conclusion}

It is worth to consider the inherent relationship between TCM and hypnotic rituals of AUCHC Method since AUCH(C Method could open a new window to the feasibility and acceptability of the integrative use of hypnosis and acupuncture. The hypnotic treatment protocol and the basic hypnotic rituals used in AUCH@ Method have been developed spontaneously after many years of clinical experiences and observations of the first author in the search of enhancing the hypnotic experience and increasing the efficiency of the treatment. On the other hand, it is possible to examine the efficiency of AUCH(C) Method by considering its applications in minor and major surgical operations or other painful or anxiety provoking medical or surgical interventions besides its various effective applications in different fields of medicine. AUCH@ can be applied as a valuable tool to prepare the patient for surgery both mentally and physiologically, to maintain the stability of vital signs and retain proper homeostasis during intraoperative period, and to facilitate postoperative healing. Furthermore, AUCH (C) can be used as the sole anesthetic for surgery by controlling the physical stimulus of pain and also the emotional response to pain such as anxiety, muscle tension, and apprehension [16-20]. On the other side, further research is necessary to elaborate the mechanism and rationale behind the inherent relationship between TCM and hypnotic rituals of AUCHC Method, and as a result to offer further understanding regarding the integrative use of hypnosis and acupuncture.

\section{Authors}

All research done by the authors.

\section{Financial Support}

This study did not receive any specific grant from funding agencies in the public, commercial, or not-for-profit sectors.

\section{Conflict of Interest}

The authors declare that they have no conflicts of interest.

\section{References}

1. Adachi T, Fujino H, Nakae A, Mashimo T, Sasaki J (2014) A Meta-Analysis of hypnosis for chronic pain problems: a comparison between hypnosis, standard care, and other psychological interventions. International Journal of Clinical and Experimental Hypnosis 62(1): 1-28.

2. Flammer E, Bongartz W (2003) On the efficacy of hypnosis: a metaanalytic study. Contemporary Hypnosis 20: 179-197.

3. Montgomery GH, Duhamel KN, Redd WH (2000) A meta-analysis of hypnotically induced analgesia: how effective is hypnosis? International Journal of Clinical and Experimental Hypnosis 48(2): 138-153.

4. Montgomery GH, David D, Winkel G, Silverstein JH, Bovbjerg DH (2002) The effectiveness of adjunctive hypnosis with surgical patients: a metaanalysis. Anesthesia and analgesia 94(6): 1639-1645.

5. Shih M, Yang Y-H, Koo M (2009) A Meta-Analysis of hypnosis in the treatment of depressive symptoms: a brief communication. International Journal of Clinical and Experimental Hypnosis 57(4): 431-442.

6. Barabasz A, Watkins JG (2005) Hypnotherapeutic techniques. $\left(2^{\text {nd }}\right.$ edn), Taylor \& Francis, New York, USA.

7. Schiff E, Gurgevich S, Caspi $O$ (2007) Potential synergism between hypnosis and acupuncture-is the whole more than the sum of its parts? Evid Based Complement Alternat Med 4(2): 233-240.

8. Aung SKH, Chen WPD (2011) Clinical introduction to medical acupuncture. Thieme (1st $\mathrm{edn})$, pp. 336.

9. Hecker HU (2005) Practice of Acupuncture: Point Location, Treatment Options, TCM Basics. Thieme

10. Lu DP, Lu GP, Kleinman L (2001) Acupuncture and Clinical Hypnosis for Facial and Head and Neck Pain: A Single Crossover Comparison. American Journal of Clinical Hypnosis 44: 141-148.

11. Zeltzer LK, Tsao JCI, Stelling C, Powers M, Levy S, et al. (2002) A Phase I Study on the Feasibility and Acceptability of an Acupuncture/Hypnosis Intervention for Chronic Pediatric Pain. Journal of pain and symptom management 24(4): 437-446. 
12. Quirico PE, Pedrali T (2007) Teaching Atlas of Acupuncture: Channels and Points. Thieme

13. Lian YL, Ogal HP, Stör W (2005) The Pictorial Atlas of Acupuncture: An Illustrated Manual of Acupuncture Points. Könemann.

14. Öztürk AÖ (2004) AUCH@ (Awareness under Conscious Hypnosis) Method for Total Hysterectomy. In Proceedings of the $16^{\text {th }}$ ISH (International Society of Hypnosis) Congress: Hypnosis and Hypnotherapy, Singapore.

15. Öztürk AÖ, Öztürk G (2016) The relationship of Awareness Under Conscious Hypnosis (AUCH(C) method with Yamamoto New Scalp Acupuncture (YNSA) and Soliman's Hand Acupuncture. European Journal of Integrative Medicine 8, Supplement 1: 35-36.

16. Öztürk AÖ (2014) The Relationship of AUCH Hypnosis and Mind-Body Medicine. Acupuncture \& electro-therapeutics research 39: 413.
17. Öztürk AÖ (2013) Retrospective Evaluation of Hypnoanesthesia Operations in Turkiye with AUCHC (Awareness under Conscious Hypnosis) Method between 1971-2013. In Proceedings of the $1^{\text {st }}$ International Conference on Hypnosis in Medicine, Budapest, Hungary.

18. Öztürk AÖ (2014) Hypnosis as an Integrative Medicine Method and a Case Study about Ob/Gyn Operation under Hypnosis. Acupuncture \& electro-therapeutics research 39: 403-404.

19. Öztürk AÖ (2006) Using Hypnosis in a Case of Cholecystectomy, a Case Report. European Journal of Pain 10(S1): S226.

20. Öztürk AÖ (2005) AUCH Method Applied in Surgery. Hypnos: Swedish Journal of Hypnosis in Psychotherapy and Psychosomatic Medicine and the Journal of European Society of Hypnosis in Psychotherapy and Psychosomatic Medicine 32: 139-143.

\section{Your next submission with Juniper Publishers will reach you the below assets}

- Quality Editorial service

- Swift Peer Review

- Reprints availability

- E-prints Service

- Manuscript Podcast for convenient understanding

- Global attainment for your research

- Manuscript accessibility in different formats ( Pdf, E-pub, Full Text, Audio)

- Unceasing customer service

Track the below URL for one-step submission https://juniperpublishers.com/online-submission php 